\title{
A Method for Detection of Markers for Epithelial-Mesenchymal Transition based on Single Cell Transcriptomic Data
}

\author{
Kosho Murayama \\ Graduate School of Information Science and Technology, \\ Osaka University \\ Suita, Japan
}

\begin{abstract}
Biomarker detection has been crucially important to understand the biology and mechanism of epithelial-mesenchymal transition (EMT) related to tumor progression and treatment resistance. The existing method in a previous study simply detected differentiallyexpressed genes by ranking all genes by their variances. This paper proposes a novel method to detect markers for respective lineages in the EMT process. Our method consists of two steps: first, inference trajectories to identify the lineages of transitional processes along with the epithelial-mesenchymal transition, and secondly, identify markers of both EMT progression and reversion lineages with differential expression analysis. Using this method, we successfully detected potential markers related to EMT, Phospholipid phosphatase 4 (PLPP4), which have not been detected by the existing method.
\end{abstract}

\section{CCS CONCEPTS}

- Applied computing $\rightarrow$ Computational transcriptomics.

\section{KEYWORDS}

Marker detection, Pseudotime analysis, Single-cell RNA transcriptome, Epithelial-mesenchymal transition

\section{ACM Reference Format:}

Kosho Murayama and Hideo Matsuda. 2022. A Method for Detection of Markers for Epithelial-Mesenchymal Transition based on Single Cell Transcriptomic Data. In 2022 12th International Conference on Bioscience, Biochemistry and Bioinformatics (ICBBB '22), Fanuary 7-10, 2022, Tokyo, Japan. ACM, New York, NY, USA, 6 pages. https://doi.org/10.1145/3510427.3510436

\section{INTRODUCTION}

In cancer research, the search for changes in characteristics associated with the evolution of cancer is very important for considering the next therapeutic strategy. Epithelial-mesenchymal transition (EMT) is a well-known and important factor in the transition in malignancy associated with tumor progression due to the evolution

\footnotetext{
${ }^{*}$ Corresponding author

Permission to make digital or hard copies of all or part of this work for personal or classroom use is granted without fee provided that copies are not made or distributed for profit or commercial advantage and that copies bear this notice and the full citation on the first page. Copyrights for components of this work owned by others than ACM must be honored. Abstracting with credit is permitted. To copy otherwise, or republish, to post on servers or to redistribute to lists, requires prior specific permission and/or a fee. Request permissions from permissions@acm.org.

ICBBB '22, January 7-10, 2022, Tokyo, Japan

(C) 2022 Association for Computing Machinery.

ACM ISBN 978-1-4503-8738-5/22/01...\$15.00

https://doi.org/10.1145/3510427.3510436

of cancer and is also considered to be one of the causes of drug
}

\author{
Hideo Matsuda* \\ Graduate School of Information Science and Technology, \\ Osaka University \\ Suita, Japan \\ matsuda@ist.osaka-u.ac.jp
}

resistance [8]. In recent years, single-cell RNA-seq analysis for evaluating gene expression at the single-cell level has been developed and attracted attention. This analysis can elucidate the characteristics of individual cells and molecules and is a powerful software tool for promoting a more comprehensive understanding. Since single-cell RNA-seq analysis requires handling enormous amounts of gene expression data from respective single cells, studies on more effective analysis approaches have been studied to date.

In these studies, clustering methods that define cell types in samples such as Seurat [14], pseudotime constructed from similarities in gene expression between cells and infer the trajectory (for example Monocle [11] and slingshot [13]) for estimating gene expression over time in response to cell development and stimulus reactions [9], etc. Based on the complex processes of EMT, single-cell approaches have been applied to the field of EMT for elucidating the mechanism recently. For example, for identifying the EMT-related markers, clustering and pseudotime analysis are conducted by Monocle as a semi-supervised approach [10]. Also, an unsupervised learning method (QuanTC) is proposed to infer and quantify the transitional property of individual cells in scRNA-seq data [12]. By applying the method to the analysis of the transition trajectories from EMT single-cell datasets, they analyze transition cells and dynamics of EMT that highlight the transition trajectories mediated by intermediate cell states. In our previous study, we identify markers that are differentially expressed as specific lineages progress toward drug resistance by combining clustering, trajectory inference, and gene expression analysis of each lineage [7]. Another single-cell-based approach to use mass cytometry time-course analysis resolves lung cancer EMT states through TGF $\beta$-treatment and identifies through TGF $\beta$-withdrawal, a distinct MET state [4]. In this paper, we propose a novel method for analyzing EMT processes. The objective of our study is to identify markers expressing along the transition process through EMT.

\section{PREVIOUS STUDY}

A previous study [1] obtains single-cell RNA-seq data from four different cancer cell lines capable of undergoing an EMT (A549, lung; DU145, prostate; MCF7, breast; and OVCA420, ovarian). These cell lines were exposed each to known EMT-inducing factors TGFB1, EGF, and TNF over 12 distinct EMT time-course experiments. For each of the 12 conditions, samples were collected at five distinct time points from 8 hours to 1 week ("0d", " $8 \mathrm{~h} "$, " $1 \mathrm{~d} "$, "3d", and "7d") after treatment, and three additional time points from 8 hours to 3 days ("7d+8h off", "7d+1d off", and "7d+3d off") after the EMT-inducing stimulus had been removed as shown in Figure 1. These data have been deposited in the NCBI Gene Expression Omnibus under the 


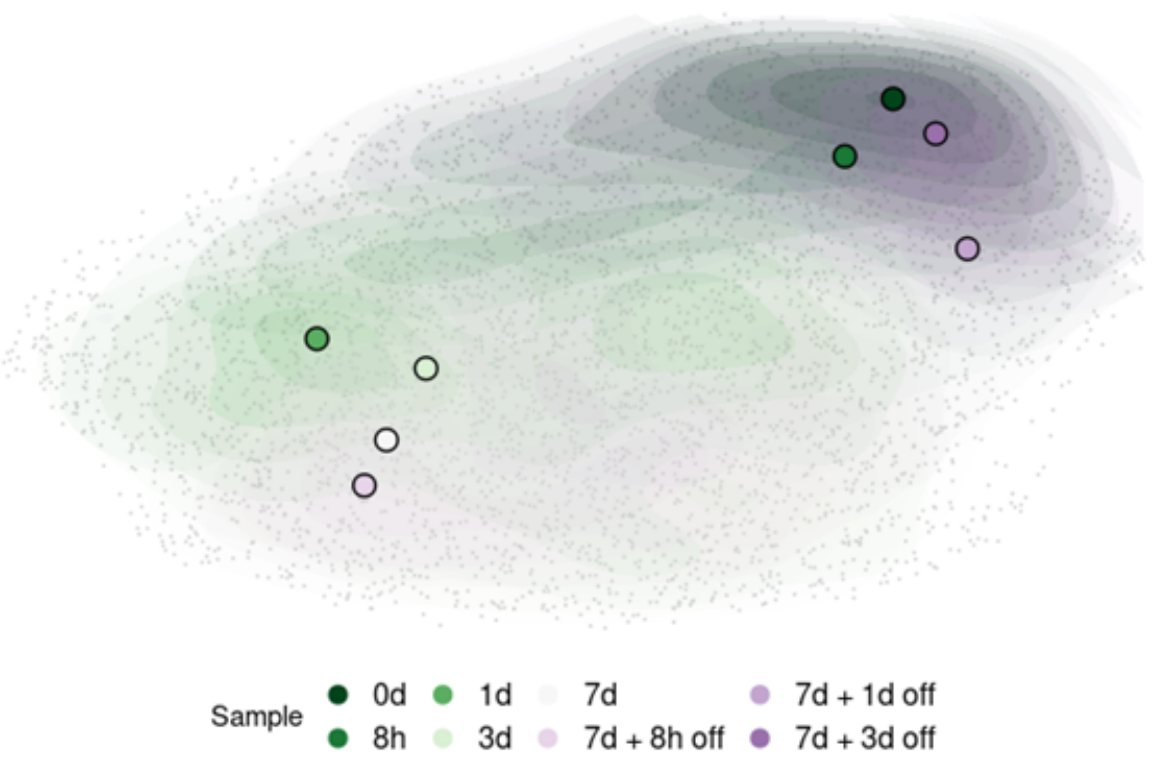

Figure 1: UMAP embeddings of TNF stimulated DU145 cells in the time-course experiments (reconstructed from the data in $[1])$.

accession GSE147405. They assessed the temporal progression of each above time course by using the R package Seurat [14] and evaluated gene expression dynamics throughout the pseudotime by using the R package psupertime [16]. While the cells were arranged along with the physical time points of the obtained cell samples and they pseudotemporally ordered the cells from each condition, the previous study did not fully perform trajectory inference but computed pseudotime along the paths connecting the centroids of the successive time-course experiment data. In addition, since they omitted the treatment withdrawal samples, only EMT progression data were used to assess changes in gene expression along with a pseudotime series.

\section{MARKER ANALYSIS FOR EMT}

\subsection{Lineage Analysis}

Our method consists of three steps: (1) perform trajectory inference to identify lineages of transitional processes along with the EMT by minimum spanning tree (MST), (2) identify the lineage for EMT reversion in addition to EMT progression, and (3) detect markers for both of the EMT progression and reversion lineages with differential expression analysis. By taking advantage of this method, we can identify markers that exhibit differentially expressed along the specific lineages of EMT.

We conducted trajectory inference with slingshot [13]. In the inference with slingshot, we set "0d" as a start cluster (green circle) and "7d" as an end cluster (red circle) in the EMT progression. In addition, we set "7d+8h" as a start cluster (green circle) and "7d+3d" as an end cluster (red circle) in the EMT reversion. We detected respective lineages by the inference in the case of DU145 prostate cells stimulated by TNF as shown in Figures 2 and 3. These lineages denote the temporal changes from epithelial to mesenchymal in the EMT progression and from mesenchymal to epithelial in the
EMT reversion, respectively through the comparison of temporal dynamics of the EMT, pseudotemporally ordered the cells for each condition. We also detected each lineage in the other conditions (data not shown).

\subsection{Detecting markers along both EMT progression and reversion lineages}

Given the lineages identified in Section 3.1, we performed statistical tests on gene expression along the lineages by using tradeSeq [2]. To extract markers for the EMT, we assessed differential expression between the starting and ending points along the lineages of the EMT progression and reversion. We used the startVsEndTest function in tradeSeq [2] as a Wald test to assess the null hypothesis that the average expression at the starting point is equal to the average expression at the endpoints. We detected statistically significant markers in both of the EMT progression and reversion lineages. Table 1 shows the top five markers of the EMT progression and reversion lineages for TNF stimulated DU145 cells detected by the test. Interestingly, Interleukin (IL)-32 and PLPP4 were detected as one of the statistically important markers in both of the two lineages. Figures 4 and 5 show the continuous gene expression of IL-32 and PLPP4, respectively. Furthermore, TGFB1 stimulated OVCA420 cells showed a similar trend about IL-32 in both the lineages of the EMT progression and regression (data not shown). These results may reflect that IL-32 and PLPP4 have a significant role in the EMT in these conditions.

Moreover, we evaluated the association of markers between the EMT progression and reversion for TNF stimulated DU145 cells by Venn diagrams. We adapted the MSigDB hallmark EMT gene set as a reference of canonical EMT-related genes [5]. We identified TAGLN, LOX, VEGFC, LAMC2, TNFAIP3 in addition 


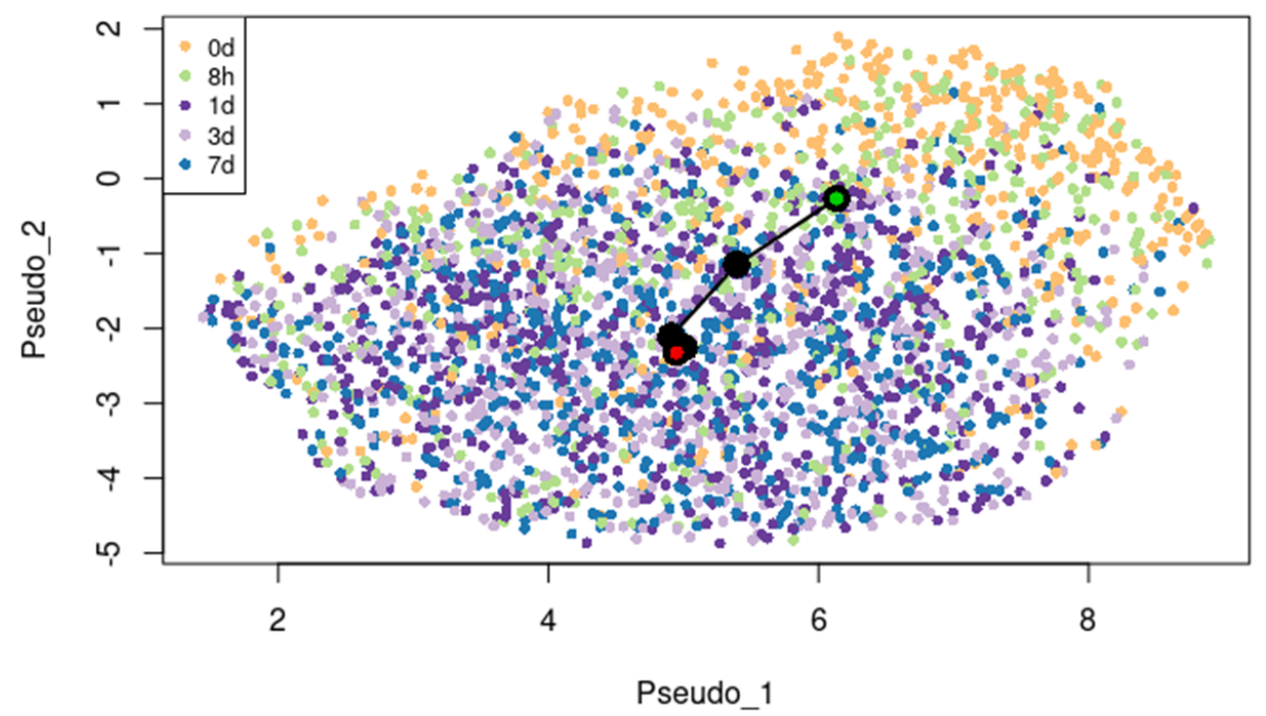

Figure 2: Lineage of the EMT progression detected by trajectory inference for TNF stimulated DU145 cells.

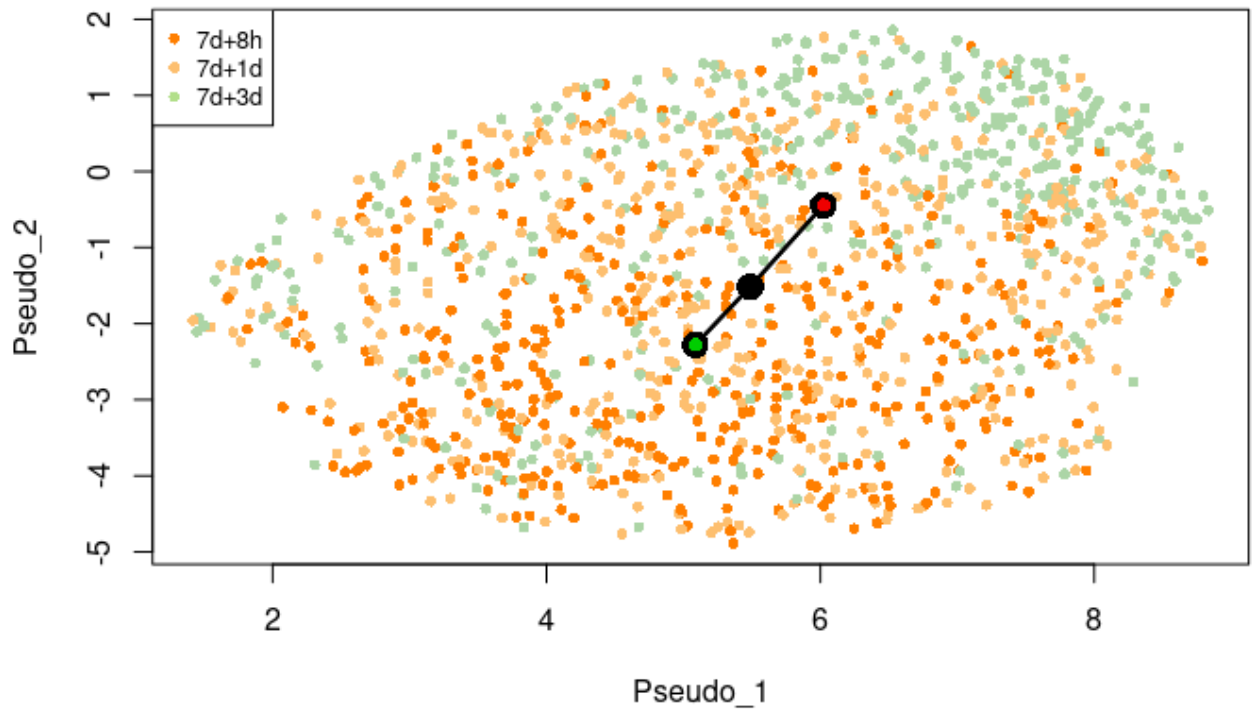

Figure 3: Lineage of the EMT reversion detected by trajectory inference for TNF stimulated DU145 cells.

to IL-32 in both the EMT progression and reversion in Figure 6. Also, we identified ADAM12, TNC, and GADD45A in only the EMT progression and CAPG and ITGA2 in only the EMT reversion. In this analysis, the concordance rate with the EMT hallmark genes was a part as shown in Figure 6. As the reason that the rate is low, the previous study reports that the EMT process is vastly contextspecific, with only a small number of response genes being shared in any two conditions [1]. If we would extend to apply our analysis to the other conditions, we could detect more different markers that would be additionally shared with the EMT hallmark genes. Another reason is that we used the startVsEndTest function to analyze the differential expression between only the start and end points of a lineage. For this reason, we cannot detect potential markers that exhibit significant changes within a lineage, e.g., early driver genes that affect the EMT process only at its early stage of the process. We will extend our method to the detection of such EMT markers.

\subsection{Comparison with the previous study}

We compared the markers described in Section 3.2 to the differentiallyexpressed markers in the previous study by psupertime $[1,16]$. The genes including IL-32, ITGA2, KLF6, OCIAD2, PLAU, KRT19 that were differentially expressed as the upregulated genes in the previous study were also detected as EMT-relevant genes by our 
Table 1: Markers detected along the EMT progression and reversion lineages of TNF stimulated DU145 cells.

\begin{tabular}{|c|c|c|}
\hline \multicolumn{2}{|c|}{ EMT progression lineage } & logFClineage \\
\hline IL32 & 84.93 & 1.84 \\
\hline PLPP4 & 48.60 & 2.53 \\
\hline FHL2 & 44.62 & 1.42 \\
\hline LTB & 40.01 & 2.29 \\
\hline SFTA1P & 38.78 & 1.28 \\
\hline \multicolumn{3}{|c|}{ EMT reversion lineage } \\
\hline & waldStat_lineage & logFClineage \\
\hline ISG15 & 76.76 & -2.14 \\
\hline IL32 & 46.32 & -2.02 \\
\hline ISG20 & 39.13 & -1.47 \\
\hline PLPP4 & 24.66 & -2.72 \\
\hline WFDC2 & 22.79 & -2.10 \\
\hline
\end{tabular}
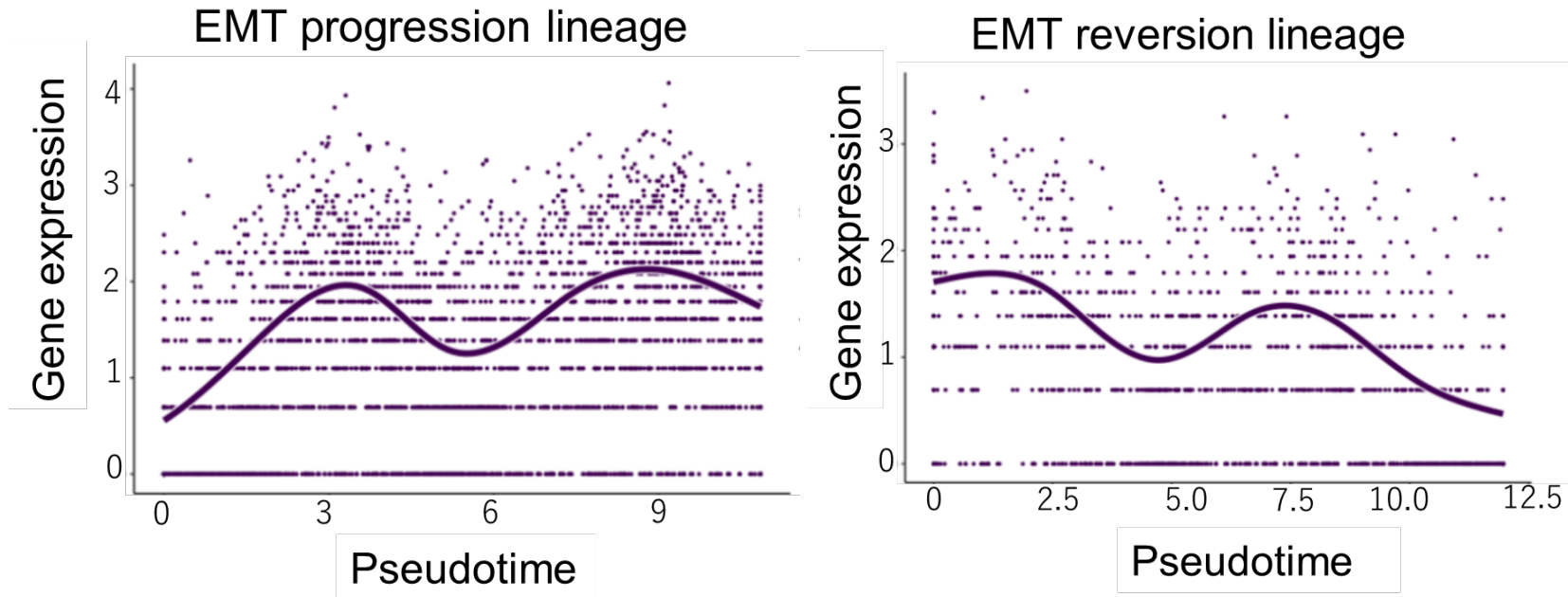

Figure 4: Expression of IL-32 along the two lineages.

method. IL32 and ITGA2 were included in the EMT hallmark genes set. When we confirmed the individual pseudotime of the genes that were not detected by our approach among the genes listed in the previous studies, the expression level was constant and no change was observed(data not shown). Interestingly, PLPP4 was not included in the resulting list of the previous study (data not shown). This suggests that our detection of markers along lineage can provide a different means for understanding EMT.

\section{DISCUSSION}

To elucidate the biology of EMT is one of the key research fields for considering the next cancer treatment. Currently, lots of researchers have been trying to develop a more effective approach to identify gene expression markers by the huge amount of data from bulk RNA-seq and single-cell RNA-seq to identify the key factor of EMT. In this paper, we conducted a lineage-based differential expression analysis to identify markers that exhibit differential expression as specific lineages in the EMT.
As a result of the markers detected in the EMT, IL32 was identified as both of the EMT progression and reversion in the conditions of TNF stimulated DU145 cells and TGFB1 stimulated OVCA420 cells. IL-32 is a well-known cytokine associated with inflammation, autoimmune disease, virus infections, and cancer [3]. In especially, Cancer-associated fibroblasts (CAF) secreted IL32 promotes breast cancer cells invasion and metastasis through the enhancement of the EMT markers expression to promote tumor invasion [15].

Moreover, PLPP4 was identified as both of the EMT progression and reversion in the conditions of TNF stimulated DU145 cells. PLPP4 has the potential for the proliferation and tumorigenesis of lung carcinoma by promoting cell cycle progression [17]. High expression of PLPP4 is positively associated with advanced clinicopathological features and correlates with poor survival in lung carcinoma patients [17]. Furthermore, PLPP4 was identified as one of the key genes to promote bowel metastases among patients with ovarian cancer [6]. While PLPP4 has not been reported as one of 

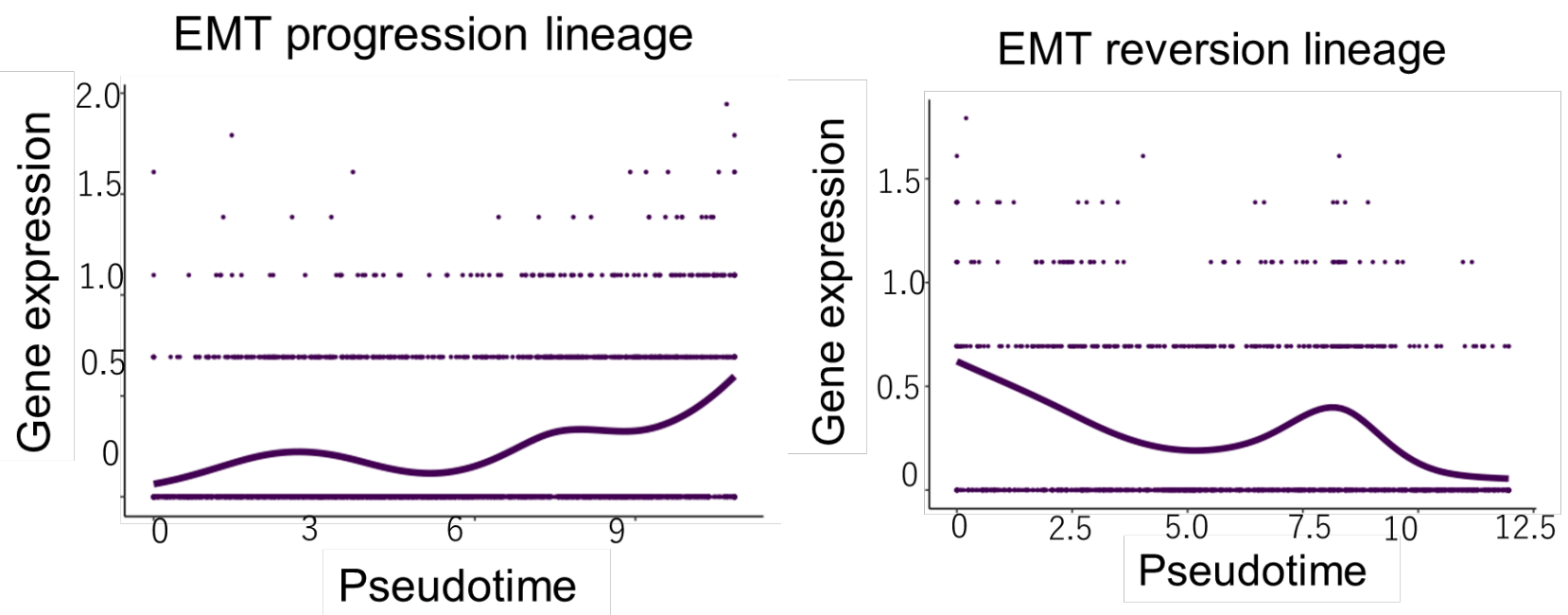

Figure 5: Expression of PLPP4 along the two lineages.

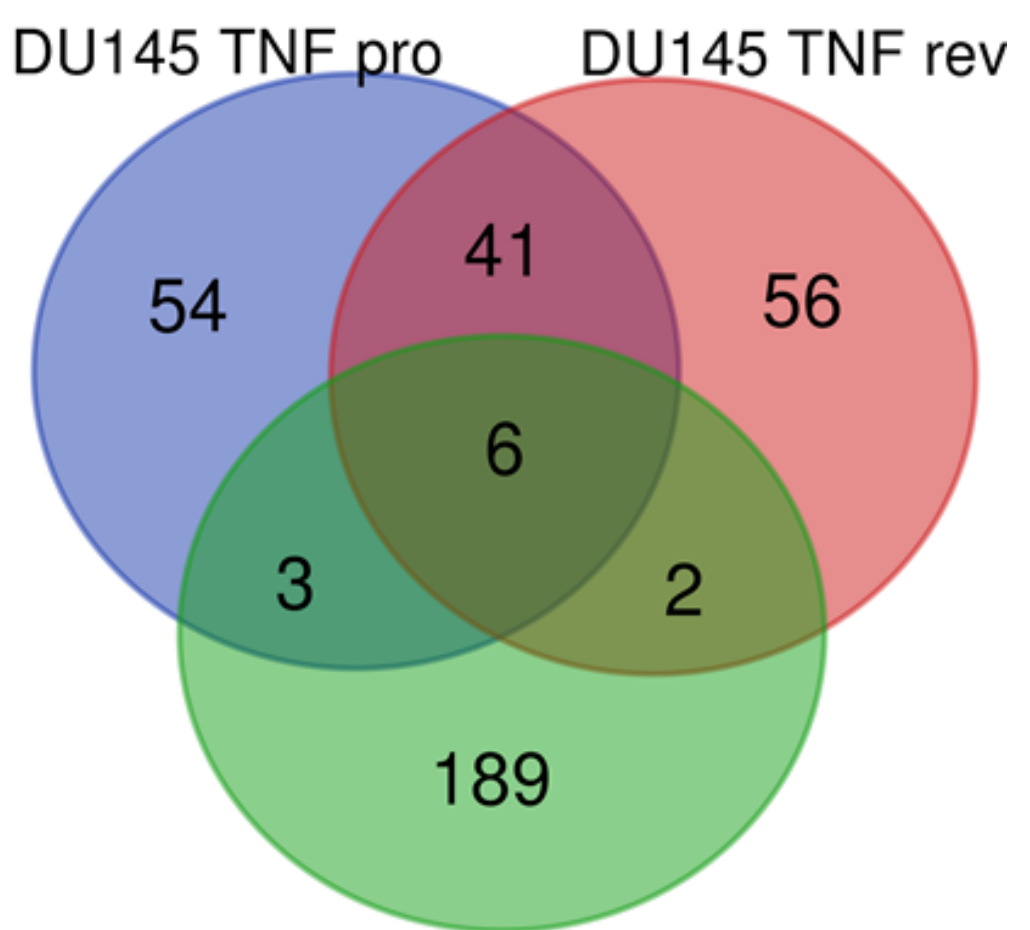

EMT hallmark genes

Figure 6: Comparison of the top markers between the EMT progression and reversion for TNF stimulated DU145 cells with the EMT hallmark genes.

the EMT-related genes so far, these reports suggest that PLPP4 contributes to the EMT in addition to the tumor progression.

\section{CONCLUSION}

In this paper, we propose a novel method for the detection of markers related to the EMT. The previous study [1] did not fully perform trajectory inference but computed pseudotime along the paths connecting the centroids of the successive time-course experiment data. Our method could evaluate continuous state transitions after 
the administration of EMT stimulating and inducing factors based on single-cell transcriptomic data, and it successfully detected a potential marker, PLPP4, which was not detected by the previous study. This lineage-specific marker analysis will provide an efficient strategy to further understand the processes of changes in cell state during the EMT through the combination of the existing methods. Our future works aim at developing a more effective approach including the creation of new tools that can identify more potential and meaningful markers related to the improvement of trajectory inference methods.

\section{ACKNOWLEDGMENTS}

This work was supported in part by JSPS KAKENHI Grant Numbers JP18H04124, JP19K22894, JP20H04947, and JP21K19827 Japan.

\section{REFERENCES}

[1] David P Cook and Barbara C Vanderhyden. 2020. Context specificity of the EMT transcriptional response. Nature Communications 11, 2142 (2020). https //doi.org/10.1038/s41467-020-16066-2

[2] Koen Van den Berge, Hector Roux de Bézieux, Kelly Street, Wouter Saelens, Robrecht Cannoodt, Yvan Saeys, Sandrine Dudoit, and Lieven Clement. 2020 Trajectory-based differential expression analysis for single-cell sequencing data. Nature Communications 11, 1 (2020), 1201. https://doi.org/10.1038/s41467-02014766-3

[3] Anuradha Gautam and Bhaswati Pandit. 2021. IL32: The multifaceted and unconventional cytokine. Human Immunology 82 (2021), 659-667. Issue 9. https://doi.org/10.1016/j.humimm.2021.05.002

[4] Loukia G. Karacosta, Benedict Anchang, Nikolaos Ignatiadis, Kimmey Samuel C, Benson Jalen A, Shrager Joseph B, Robert Tibshirani, Bendall Sean C, and Plevritis Sylvia K. 2019. Mapping lung cancer epithelial-mesenchymal transition states and trajectories with single-cell resolution. Nature Communications 10, 5587 (2019). https://doi.org/10.1038/s41467-019-13441-6

[5] Arthur Liberzon, Chet Birger, Helga Thorvaldsdóttir, Mahmoud Ghandi, Jill P Mesirov, and Pablo Tamayo. 2015. The Molecular Signatures Database (MSigDB) hallmark gene set collection. Cell Systems 1 (2015), 417-425. Issue 6. https: //doi.org/10.1016/j.cels.2015.12.004

[6] Andrea Mariani, Chen Wang, Ann L Oberg, Shaun M Riska, Michelle Torres, Joseph Kumka, Francesco Multinu, Gunisha Sagar, Debarshi Roy, Deok-Beom Jung, Qing Zhang, Tommaso Grassi, Daniel W Visscher, Vatsal P Patel, Ling Jin, Julie K Staub, William A Cliby, Saravut J Weroha, Kimberly R Kalli, Lynn C Hartmann, Scott H Kaufmann, Ellen L Goode, and Viji Shridhar. 2019. Genes associated with bowel metastases in ovarian cancer. Gynecologic Oncology 154 (2019), 495-504. Issue 9. https://doi.org/10.1016/j.ygyno.2019.06.010
[7] Kosho Murayama and Hideo Matsuda. 2021. Detecting Lineage-Specific Marker Genes for Tumor Evolution Based on Single Cell Transcriptome. International fournal of Bioscience, Biochemistry and Bioinformatics 11, 3 (2021), 50-57. https: //doi.org/10.17706/ijbbb.2021.11.3.50-57

[8] M.Angela Nieto, Ruby Yun-Ju Huang, Rebecca A. Jackson, and Jean Paul Thiery. 2016. EMT: 2016. Cell 166, 1 (2016), 21-45. https://doi.org/10.1016/j.cell.2016.06. 028

[9] Naoki Osato, Hironori Shigeta, Shigeto Seno, Yutaka Uchida, Junichi Kikuta, Masaru Ishii, and Hideo Matsuda. 2019. Single-cell Transcriptome Analysis of Mouse Leukocytes in Inflammatory Stimulation. 2019 IEEE International Conference on Bioinformatics and Biomedicine (BIBM) (2019), 1229-1231. https: //doi.org/10.1109/BIBM47256.2019.8983394

[10] Ievgenia Pastushenko, Audrey Brisebarre, Alejandro Sifrim, Marco Fioramonti, Tatiana Revenco, Soufiane Boumahdi, Alexandra Van Keymeulen, Daniel Brown, Virginie Moers, Sophie Lemaire, Sarah De Clercq, Esmeralda Minguijón, Cédric Balsat, Youri Sokolow, Christine Dubois, Florian De Cock, Samuel Scozzaro, Federico Sopena, Angel Lanas, Nicky D’Haene, Isabelle Salmon, Jean-Christophe Marine, Thierry Voet, Panagiota A. Sotiropoulou, and Cédric Blanpain. 2018. Identification of the tumour transition states occurring during EMT. Nature 556 (2018), 463-468. https://doi.org/10.1038/s41586-018-0040-3

[11] Xiaojie Qiu, Qi Mao, Ying Tang, Li Wang, Raghav Chawla, Hannah A Pliner, and Cole Trapnell. 2017. Reversed graph embedding resolves complex single-cell trajectories. Nature Methods 14, 10 (2017), 979-982. https://doi.org/10.1038/ nmeth. 4402

[12] Yutong Sha, Shuxiong Wang, Peijie Zhou, and Qing Nie. 2020. Inference and multiscale model of epithelial-to-mesenchymal transition via single-cell transcriptomic data. Nucleic Acids Research 48 (2020), 9505-9520. Issue 17. https://doi.org/10.1093/nar/gkaa725

[13] Kelly Street, Davide Risso, Russell B. Fletcher, Diya Das, John Ngai, Nir Yosef, Elizabeth Purdom, and Sandrine Dudoit. 2018. Slingshot: cell lineage and pseudotime inference for single-cell transcriptomics. BMC Genomics 19, 1 (2018), 477. https://doi.org/10.1186/s12864-018-4772-0

[14] Tim Stuart, Andrew Butler, Paul Hoffman, Christoph Hafemeister, Efthymia Papalexi, William M. Mauck, Marlon Stoeckius, Peter Smibert, and Rahul Satija. 2019. Comprehensive integration of single cell data. Cell 177 (2019), 18881902.e21. Issue 7. https://doi.org/10.1016/j.cell.2019.05.031

[15] Siyang Wen, Yixuan Hou, Lixin Fu, Lei Xi, Dan Yang, Maojia Zhao, Yilu Qin, Kexin Sun, Yong Teng, and Manran Liu. 2019. Cancer-associated fibroblast (CAF)-derived IL32 promotes breast cancer cell invasion and metastasis via integrin $\beta 3$-p38 MAPK signalling. Cancer Letters 442 (2019), 320-332. https: //doi.org/10.1016/j.canlet.2018.10.015

[16] Macnair Will and Claassen Manfred. 2019. Psupertime: Supervised pseudotime inference for single cell RNA-seq data with sequential labels. bioRxiv (2019). https://doi.org/10.1101/622001

[17] Xin Zhang, Lan Zhang, Bihua Lin, Xingxing Chai, Ronggang Li, Yuehua Liao, Xinghui Deng, Qiongru Liu, Wenli Yang, Yubo Cai, Wei Zhou, Zhichao Lin, Wenhai Huang, Meigong Zhong, Fangyong Lei, Jinhua Wu, Shuaishuai Yu, Xiaoping Li, Shangren Li, Yueyue Li, Jincheng Zeng, Wansheng Long, Dong Ren, and Yanming Huang. 2017. Phospholipid Phosphatase 4 promotes proliferation and tumorigenesis, and activates Ca 2+-permeable Cationic Channel in lung carcinoma cells. Molecular Cancer 16 (2017), 147. https://doi.org/10.1186/s12943-017-0717-5 